\title{
PROGRAMA "UEPG ABRACCA": UMA PERSPECTIVA INTERDISCIPLINAR SOBRE A SAÚDE MENTAL NA UNIVERSIDADE
}

\section{"UEPG ABRACA" PROGRAM: AN INTERDISCIPLINARY PERSPECTIVE ON MENTAL HEALTH AT THE UNIVERSITY}

\author{
Lara Simone Messias Floriano* \\ ORCID: https://orcid.org/0000-0003-4801-2767 \\ Alessandra Rodrigues Martins
ORCID:
*ttps://orcid.org/0000-0001-8328-1298 \\ Caroline Vezine Brabicoski*** \\ ORCID: https://orcid.org/0000-0002-5074-7191 \\ Amanda de Mello Silva Rodrigues*** \\ ORCID:https://orcid.org/0000-0003-1147-9259
}

\section{RESUMO:}

Este artigo discorre sobre as atividades desenvolvidas pelo serviço de atenção psicossocial Programa "UEPG Abraça", da Universidade Estadual de Ponta Grossa, e relata a perspectiva da equipe multiprofissional sobre o processo de trabalho. Trata-se de estudo descritivo sobre as atividades do Programa "UEPG Abraça" e relato da equipe sobre a atuação interdisciplinar desempenhada no periodo entre fevereiro a setembro de 2019. Realizou-se entrevista semiestruturada e análise de conteúdo dos dados coletados. O referido Programa oferece acompanhamento psicossocial à comunidade universitária à luz da interdisciplinaridade. Para tanto, ressaltam-se aspectos importantes, como seguridade ofertada pela rede de profissionais, ganho ao indivíduo atendido e troca de experiências. No entanto, desafios como a alta demanda de trabalho e escassez de tempo para gestão de casos precisam ser superados. Constata-se que o acompanhamento psicossocial realizado por meio de ações interdisciplinares é de extrema importância para os integrantes e principalmente ao manejo da Saúde Mental na Universidade.

Palavras chave: Saúde mental; Serviços de saúde em universidades; Sistemas de apoio psicossocial; Práticas interdisciplinares.

\begin{abstract}
:
This paper reports the activities performed in the psychosocial assistance "Programa UEPG Abraca" of the State University of Ponta Grossa and describes the multi-professional team's perspective on the work process. A descriptive study was developed on the activities of the Programa UEPG Abraça including a team's report on the interdisciplinary performance in the period between February and September 2019. A semi-structured interview and content analysis of the collected data were carried out. The Program offers psychosocial attendance to the university community based on interdisciplinarity. To this end, important aspects are highlighted such as the security offered by the professionals' network, gains to the individuals served and experience sharing. However, challenges such as high demand of work and time scarcity to have meetings focusing on case management need to be overcome. The psychosocial service performed through interdisciplinary actions is extremely important for the participants and especially for the Mental Health management at the University.
\end{abstract}

Keywords: Mental health; University health services; Psychosocial support systems; Interdisciplinary practices.

\footnotetext{
${ }^{*}$ Professora da Universidade Estadual de Ponta Grossa (UEPG), Ponta Grossa - PR, Brasil. E-mail: larasmessias@gmail.com

${ }^{* *}$ Aluna de Mestrado da Universidade Estadual de Ponta Grossa (UEPG), Ponta Grossa - PR, Brasil. E-mail: aleeh_martins@hotmail.com ${ }^{* * *}$ Enfermeira do Hospital Universitário Regional dos Campos Gerais (HURCG), Ponta Grossa - PR, Brasil. E-mail: carolvezine@hotmail.com

${ }^{* * * *}$ Assistente Social do Hospital Universitário Regional dos Campos Gerais (HURCG), Ponta Grossa - PR, Brasil. Email: amsrodrigues@uepg.br
} 


\section{Introdução}

No atual contexto universitário, a Saúde Mental é um tema bastante comentado. Estudos apontam a alta prevalência de transtornos mentais entre acadêmicos e ressaltam que tais índices são superiores neste público em detrimento da população geral (CONCEIÇÃO et al., 2017; COSTA et al., 2017). As elevadas taxas são atribuídas a fatores estressores comumente presentes no cotidiano de discentes, tais como: necessidade de longas horas de estudos, cobranças quanto ao desempenho acadêmico, aquisição de novas responsabilidades e o desbravamento de um mundo diante do qual, por vezes, os indivíduos sentem-se pouco preparados para enfrentar (LANTYER et al., 2018).

A sensação de sobrecarga e "ter que dar conta de tudo" usualmente presente nos discursos dos estudantes colabora com a instalação do transtorno mental, sentimento de impotência e torna-se um empecilho para a resolução de problemas. A combinação desses fatores pode ser desencadeante do uso de álcool, tabaco e demais substâncias psicoativas, tornando o indivíduo mais vulnerável às situações de violência (SILVA et al., 2015; FERNANDES et al., 2018).

Diante do panorama alarmante presente em diversas universidades, estudos nacionais e internacionais destacam a necessidade de projetos de intervenção psicológica e psicoeducativa, buscando a melhoria da Saúde Mental desse segmento (KWAN et al., 2016; NOGUEIRA; SEQUEIRA, 2017; LEITE; NOGUEIRA, 2017; GULLIVER et al., 2017).

No Brasil, em 1957, houve o primeiro registro de um serviço de atenção psicossocial voltada aos estudantes universitários, denominado Serviço de Higiene Mental e Psicologia Clínica, criado por Galdino Loreto, enquanto docente da Faculdade de Medicina da Universidade Federal de Pernambuco. Ao longo da década de 60, outras universidades federais adotaram a prática, a exemplo dos serviços de Saúde Mental criados pela Universidade Federal do Rio Grande do Sul (UFRS), Minas Gerais (UFMG) e Rio de Janeiro (UFRJ) (CERCHIARI, 2004). Atualmente, estima-se que um terço das universidades públicas brasileiras possui alguma forma de atendimento psicossocial destinado à comunidade interna (FONAPRACE, 2015).

A Universidade Estadual de Ponta Grossa (UEPG) oferta cursos de graduação, pósgraduação em nível de especialização, mestrado e doutorado. O serviço de psicologia funcionava havia alguns anos de forma isolada, fornecendo consultas individuais à comunidade universitária. No entanto, em outubro de 2018, em uma iniciativa da nova gestão da Reitoria (2018-2022), criou-se o Programa de Extensão "UEPG Abraça", vinculada à PróReitoria de Extensão e Assuntos Culturais (PROEX), Pró-Reitoria de Recursos Humanos (PRORH) e em parceria com o Hospital Universitário Regional dos Campos Gerais (HURCG - UEPG), com a proposta de aperfeiçoar as atividades que já vinham sendo realizadas e incorporar outras práticas, visando à integralidade do cuidado em Saúde Mental.

Diante do exposto, o presente estudo teve como objetivos: discorrer sobre as atividades desenvolvidas pelo serviço de atenção psicossocial Programa "UEPG Abraça", da Universidade Estadual de Ponta Grossa, e relatar a perspectiva da equipe multiprofissional sobre o processo de trabalho para contribuir com a qualidade no cuidado em Saúde Mental no contexto universitário.

\section{Metodologia}

Trata-se de estudo descritivo, do tipo relato sobre as atividades desempenhadas por acadêmicos e profissionais integrantes da equipe multiprofissional do Programa de Extensão "UEPG Abraça", bem como a perspectiva deles em relação ao processo de trabalho desenvolvido interstício compreendido entre fevereiro e setembro de 2019. 
Consideradas as metodologias de observação sistemática, os relatos não apresentam necessidade de testar hipóteses, no entanto têm o intuito de estabelecer relações entre os achados pertinentes à realidade e ao embasamento teórico (DYNIEWICZ, 2009).

Neste estudo, foram realizadas entrevistas semiestruturadas com os profissionais e acadêmicos que atuam no referido Programa, contendo as seguintes questões: 1) Descreva as ações realizadas pela equipe multiprofissional do Programa "UEPG Abraça"; 2) Relate sua perspectiva a respeito do modelo e processo de trabalho presente no serviço em que atua, elencando potencialidades e desafios na realização do trabalho interdisciplinar.

Após esse processo, os dados foram agrupados e analisados mediante a aplicação da técnica de Análise de Conteúdo proposta por Bardin (MINAYO, 2014). De acordo com a autora, essa técnica prevê a análise de informações sobre o comportamento humano de modo sistemático, cuja função é trazer à luz o que há por trás dos conteúdos descritos.

Todos os sujeitos participantes foram esclarecidos sobre os objetivos, riscos e benefícios enquanto participantes desta pesquisa, a qual seguiu o estabelecido na Resolução 466/2012 do Conselho Nacional de Saúde e obteve aprovação do Comitê de Ética e Pesquisa da Universidade Estadual de Ponta Grossa, sob o parecer 3.604.737, CAAE: 21415319.4.0000.0105.

\section{Resultados}

O Programa "UEPG Abraça" conta com equipe multiprofissional composta por coordenadora enfermeira do serviço, 2 enfermeiras, 4 psicólogos, 1 assistente social e 1 psiquiatra em parceria com o HURCG - UEPG. O Programa conta, também, com 2 acadêmicos bolsistas do Curso de Bacharelado em Enfermagem da UEPG e graduandos do $5^{\circ}$ ano do Curso de Psicologia da Faculdade Santana, instituição parceira.

Este Programa de Extensão tem como objetivo realizar acolhimento e acompanhamento psicossocial a discentes, docentes e agentes universitários da UEPG e do HURCG, no que diz respeito às demandas de Saúde Mental: enfrentamento de transtornos mentais, álcool e outras drogas, prevenção e posvenção do suicídio, relações de conflitos entre professor/aluno/agente universitário, suporte a grupos vulneráveis, por meio de atendimentos individuais e em grupos nos campi de Uvaranas e Central da UEPG, e em espaços do HURCG.

Além do atendimento à comunidade interna, o Programa "UEPG Abraça" mantém parceria com a Polícia Rodoviária Federal (PRF), a qual proporciona atendimento psicossocial aos referidos policiais e periodicamente realiza eventos extensionistas sobre Saúde Mental voltados à população.

No que se refere ao atendimento prestado, todos que buscam inserção no Programa "UEPG Abraça" passam por acolhimento imediato, realizado pelos profissionais da equipe multiprofíssional, em ambiente seguro e sigiloso. Trata-se de um serviço de porta aberta, no qual o primeiro atendimento se dá por meio de procura direta de acadêmicos e servidores, ou via encaminhamento dos Departamentos e Pró-Reitoria de Assuntos Estudantis (PRAE) da UEPG.

Ao procurar o Programa "UEPG Abraça", os sujeitos são acolhidos pela equipe de enfermagem e serviço social, momento que permite a identificação de possíveis casos graves, caracterizando-se como estratégia fundamental nesse modelo, pois consiste no 
atendimento de todos os que procuram o Programa espontaneamente ou encaminhados por outros serviços. Isso é feito por meio de uma escuta qualificada da demanda espontânea por profissionais da equipe de Saúde Mental, com o objetivo de identificar a vulnerabilidade, considerando-se as dimensões subjetivas, biológicas e sociais.

Durante o acolhimento o profissional orienta, estabelece relação de vínculo e decide, em conjunto com o usuário, quais os encaminhamentos necessários para a atenção adequada ao problema segundo o fluxograma existente, cuja finalidade é ampliar e facilitar o acesso dos usuários aos serviços existentes, potencializando o conhecimento técnico e agregando resolutividade na intervenção, promovendo o vínculo e a responsabilização organizacional para com os usuários. O acolhimento é utilizado com vistas a desenvolver ações de promoção, prevenção e assistência junto aos membros da comunidade UEPG em um esforço de se estabelecer aderência entre o usuário e os serviços de saúde.

Nesse momento, também são fornecidos esclarecimentos a respeito do Programa, modalidades de atendimentos, tempo de espera, gratuidade e sigilo do serviço. Ao final da consulta de enfermagem, é realizada a avaliação do caso, elencando-se quanto ao nível de prioridade e aconselhamento sobre condutas e encaminhamentos.

A identificação da necessidade dos indivíduos em receber apoio psicossocial é de competência de todo enfermeiro generalista, uma vez que a formação na área de Enfermagem prepara o graduando para atuar no campo da Saúde Mental por meio da educação em saúde, tornando-se um importante membro da equipe. Nessa perspectiva, a enfermagem psiquiátrica, partindo dos preceitos e diretrizes pautadas na Reforma Psiquiátrica, visa observar de maneira holística o indivíduo, retirando o enfoque do transtorno mental e transportando-a para a pessoa, de modo a completar a equipe interprofissional e, junto ao sujeito, formular estratégias de superação e ou convívio com a condição (MENDES et al., 2018).

Ainda no que tange ao acolhimento no Programa "UEPG Abraça", a assistente social busca conhecer a realidade social dos indivíduos atendidos, seu contexto social, familiar e cultural. Ainda, ações junto aos sujeitos para o fortalecimento de fatores de proteção de Saúde Mental, orientações em relação a acessos a direitos, visando a ampliação da cidadania e encaminhamentos à Rede de Atenção Psicossocial (RAPS) e Assistência Social (COUTINHO; SANTOS, 2016).

Após esse acolhimento, conforme a disponibilidade de vaga, o indivíduo é contatado e encaminhado à psicoterapia. $\mathrm{O}$ apoio psicológico é formalizado por meio de contrato entre o participante e o psicólogo, e dura em média de 6 a 8 sessões, com tempo estimado de 50 minutos por sessão. Conforme avaliação do psicólogo, o usuário pode ser encaminhado para a psiquiatra do Programa ou dar continuidade ao tratamento em outros serviços pertencentes à RAPS.

A Psicologia vem como contribuição nos contextos universitários em programas de Saúde Mental para a obtenção de descarga emocional e autoconhecimento dos alunos. As demandas são variadas, como problemas familiares, de relacionamento, financeiro, acúmulo de atividades acadêmicas. Em geral, relações interpessoais têm refletido muito na produtividade estudantil, dessa forma cada caso possui uma demanda para ser trabalhada, pois cada acadêmico possui sua singularidade.

O fluxo de atendimento, bem como as atividades desenvolvidas são baseados no Manual de Saúde Mental elaborado por Saracceno, Asioli e Tognoni (2001), conforme ilustrado na Figura 1, a seguir: 
Figura 1 - Fluxo de assistência aos usuários Programa "UEPG ABRAÇA".

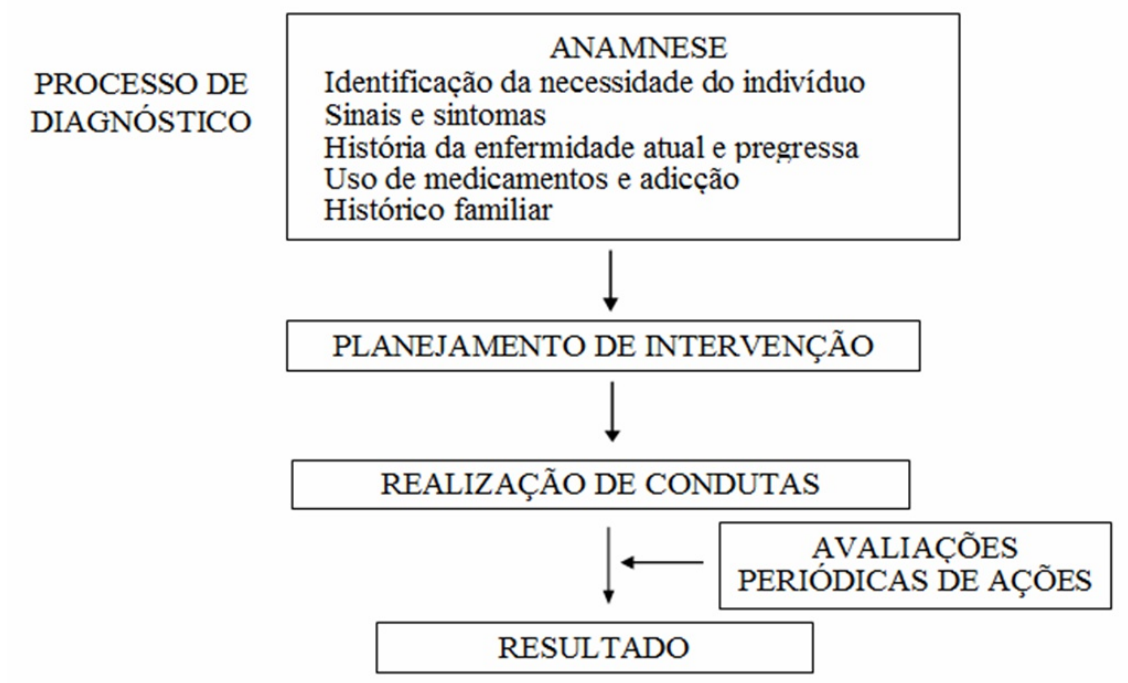

Fonte: Adaptado de SARACENO, B.; ASIOLI, F.; TOGNONI, G., 2001.

De acordo com dados levantados a partir dos registros internos do Programa, na ficha de acolhimento no primeiro semestre de 2019, cerca de 300 pessoas receberam atendimento psicossocial no Programa "UEPG Abraça", em atendimentos individuais e em grupos específicos, como o grupo terapêutico LGBTS, roda de conversa com servidores do HURCG, grupo de residentes multiprofissionais em saúde do HURCG, grupo de ansiedade e depressão, entre outros, acabando com a fila de espera que perdurava havia cerca de dois anos. Além disso, no Programa busca-se oferecer Práticas Integrativas e Complementares em Saúde (PICS) aos usuários.

Conforme a definição do Ministério da Saúde (2019), as Práticas Integrativas Complementares (PICS) são tratamentos que utilizam recursos terapêuticos baseados principalmente na Medicina Tradicional Chinesa, voltados a prevenir diversas doenças, como depressão e hipertensão, e em alguns casos também podem ser usadas como tratamentos paliativos de doenças crônicas. Consistem em um conjunto heterogêneo de práticas, produtos e saberes, agrupados pela característica comum de não pertencerem ao escopo dos conhecimentos consagrados na medicina convencional.

Streeter e colaboradores (2017), em um estudo epidemiológico que objetivou avaliar os efeitos de uma intervenção de yoga e respiração coerente em cinco respirações por minuto, em sintomas depressivos, e determinar a melhor intervenção para estudos futuros em indivíduos com transtorno depressivo, comprovam o uso de yoga e a intervenção respiratória como tratamentos voltados ao alívio dos sintomas de depressão profunda. Contudo, seus benefícios vão além, diminuindo a pressão arterial e agindo nos sistemas cardiovascular e nervoso.

Nessa perspectiva, o Programa "UEPG Abraça", em parceria com o Departamento de Educação Física da UEPG, disponibiliza 20 vagas para a prática gratuita de yoga, durante um semestre, aos indivíduos que estão em acompanhamento psicossocial. Para usufruir deste serviço, é necessário somente possuir vínculo com a UEPG e com o referido programa de extensão, bem como manter assiduidade nas aulas de yoga. Ao ofertar Práticas Integrativas Complementares aos usuários do Programa, espera-se implementar conhecimentos e práticas terapêuticas desvencilhadas da medicina tradicional, sem uso de fármacos como forma de tratamento.

Os eventos extensionistas são destaque do Programa "UEPG Abraça". Durante o ano de 2019, profissionais, acadêmicos bolsistas e voluntários do referido serviço psicossocial promoveram diversos eventos e ou participaram dos mesmos. Como 
exemplo, a acolhida do calouro que ingressa na UEPG; o evento conhecido como "Junho Branco", realizado no mês do junho de 2019, que se refere à conscientização da população sobre o uso problemático de álcool e outras drogas; que se referiram a temáticas de sobrecarga acadêmica, assédio, controle de ansiedade e estresse, mídias sociais e a influência para o pânico coletivo, desenvolvidos em parceria com a PróReitoria de Assuntos Estudantis (PRAE), Pró-Reitoria de Graduação (PROGRAD) e Instituições de Ensino Superior parceiras.

A promoção de eventos abertos à população é de grande importância, uma vez que traz à luz e ao acesso da população assuntos de extrema relevância por meio de falas e trocas de experiências de profissionais especialistas na área de Saúde mental e população em geral. Tal fato faz a aproximação do ambiente acadêmico com a comunidade externa, fortalecendo o papel social da Universidade e fomentando a formação por meio da educação dialógica (FERNANDES et al., 2016).

Outra atividade realizada pelo Programa "UEPG Abraça" é a educação para a saúde nos setores da UEPG, cujo intuito é divulgar informações sobre a Saúde Mental no âmbito universitário e as atividades desenvolvidas pelo programa, bem como firmar a corresponsabilização entre os coordenadores dos cursos da UEPG enquanto representantes do corpo docente e o programa durante as reuniões setoriais, a fim de, juntos, colaborarem para a promoção do bem-estar, Saúde Mental e reabilitação psicossocial no âmbito acadêmico.

Nessas oportunidades, levantou-se a demanda do atendimento noturno destinada aos acadêmicos que trabalham durante o dia e estudam à noite, ou moram em outras localidades distantes da Universidade. Tal iniciativa tem sido realizada no intuito de contemplar os acadêmicos que, por algum desses motivos, não possam usufruir do serviço psicossocial durante o dia e o possam fazer nesses plantões noturnos.

Atualmente, a fila de espera foi extinta em virtude do regime de plantão adotado pela equipe, no qual realizam-se atendimentos de demanda espontânea duas vezes na semana, sanando os problemas que surgem pontualmente e agilizando o processo de prestação de serviço aos indivíduos que procuram atendimento psicossocial.

Com alta procura de acadêmicos e servidores, o Programa "UEPG Abraça" pretende dobrar o número de atendimentos nos próximos anos, com a continuação de grupos de apoio com temáticas como: escuta e apoio, ansiedade, depressão, qualidade de vida no trabalho, apoio à residência multiprofissional em saúde e inserção de novos grupos.

A atuação em saúde proposta pelo Programa "UEPG Abraça", além do atendimento pontual, propõe ao indivíduo olhar para dentro de si e entender e conviver com anseios e peculiaridades, por meio da mediação do cuidado e incentivo ao autocuidado.

\section{Perspectiva da equipe sobre o modelo e processo de trabalho}

Embora o atendimento oferecido seja ordinariamente sequencial, a interdisciplinaridade é constatada durante a produção teórica, discussão de casos e grupos de apoio. Nesses momentos, ocorre uma maior interação entre os profissionais e acadêmicos participantes do Programa "UEPG Abraça" e se percebe a indissociabilidade da atuação de diferentes cuidados e olhares em Saúde Mental.

Considerada uma abordagem inovadora e padrão ouro em atendimento, a interdisciplinaridade em Saúde Mental se faz fundamental no cuidado às pessoas que se encontram em sofrimento de origem psicossocial, uma vez que os aspectos psicológicos afetam diretamente nas condições físicas e sociais do indivíduo (TREMBLAY, 2017). 
Muitos integrantes do serviço psicossocial passam por sua primeira experiência profissional e de prática acadêmica específica com a população universitária. Esse momento, permeado por anseios e inseguranças, são superados por meio da troca de conhecimentos e apoio matricial proporcionados. A experiência do trabalho interdisciplinar gera a sensação de apoio e seguridade aos integrantes e usuários conforme seus relatos. A rede firmada pela equipe corrobora o estabelecimento de vínculo entre todos os eixos do cuidado, indivíduos, familiares e profissionais (SARACENO; ASIOLI; TOGNONI, 2001).

Trabalhar com diversos profissionais da saúde é de grande relevância, pois todos possuem um único objetivo: trabalhar em prol da Saúde Mental da comunidade universitária, de modo que os sujeitos possuam um bom desempenho em suas atividades e futuramente sejam excelentes profissionais, exercendo aquilo que escolheram fazer; e, ainda, para que os agentes universitários possuam um bom rendimento, de modo a prevenir a síndrome do esgotamento profissional por exemplo.

Neste sentido, alcançar esse objetivo requer o diálogo entre os profissionais, pois ao expor de forma ética e sigilosa os casos em reuniões, consegue-se pontuar uma possível solução, como encaminhamentos e outras formas de tratamento ou atividades que possam auxiliar o universitário na boa realização de tarefas.

Dentro de uma equipe interprofissional, a comunicação deve ser estabelecida de modo efetivo em todas as etapas, seja em tomadas de decisões, no estabelecimento de condutas e resolução de problemas, para que se configure, de fato, um trabalho interdisciplinar (NASCIMENTO et al., 2017).

Não obstante o Programa "UEPG Abraça" caminhe em direção à interdisciplinaridade, alguns desafios se fazem presentes no desenvolvimento do trabalho. A alta demanda de atendimentos e a diversidade de trabalhos específicos dificultam os encontros periódicos entre os diferentes profissionais e, por conseguinte, a atuação interprofissional. Tal fato também é apontado na literatura, e aspectos como escassez de tempo para desenvolver atividades interdisciplinares, sobrecarga de trabalho, dificuldade em estabelecer dialogo efetivo e falta de interesse dos participantes configuram- barreiras a serem rompidas na implementação da interdisciplinaridade (FERRO et al., 2014).

\section{Conclusão}

Com relação à comunidade universitária, estudos mostram que docentes, discentes e técnicos podem apresentar os mais diversos transtornos mentais, como ansiedade, depressão, abuso de álcool e outras drogas, tentativas de suicídio, entre outros, o que leva à necessidade de se olhar para essa população e lhe proporcionar acolhimento, informações acerca dos enfrentamentos do dia a dia, tanto no meio universitário, quanto junto a familiares.

Em vista disso, diante da preocupação a respeito dos aspectos psicossociais que permeiam a vida universitária, o Programa "UEPG Abraça" foi criado pela gestão da Reitoria (2018-2022) para fornecer acolhimento e acompanhamento psicossocial e, assim, abarcar a necessidade iminente.

Assim, se faz necessário que seja desenvolvido um trabalho em conjunto, envolvendo a equipe de saúde, o indivíduo e sua família, priorizando a corresponsabilização dos envolvidos e pautado na interdisciplinaridade.

Como resultados, obteve-se que os aspectos positivos referentes ao trabalho interdisciplinar são a seguridade ofertada pela rede de profissionais, o ganho ao indivíduo atendido, a troca de experiências interprofissional e utilização de ferramentas de cuidado 
interdisciplinar. No entanto, desafios também foram elencados, sendo eles a sobrecarga de trabalho e dificuldade em estabelecer encontros periódicos para gestão de casos e discussões em grupo.

Diante ao exposto, percebe-se a relevância de um serviço psicossocial dessa magnitude para a reabilitação psicossocial do sujeito, bem como a importância do mesmo na Rede de Atenção Psicossocial (RAPS) do município e o impacto positivo da interdisciplinaridade para o serviço em Saúde Mental, tanto no que diz respeito a relação e o ganho interprofissional, quanto ao qualidade do atendimento prestado aos usuários do serviço. Por fim, espera-se que este estudo corrobore com a literatura sobre interdisciplinaridade em Saúde Mental no âmbito universitário, bem como incentivem a criação de mais serviços e modelos de trabalho como este.

\section{Referências}

CERCHIARI, E. Saúde mental e qualidade de vida em estudantes universitários. Tese (Doutorado) Universidade Estadual de Campinas - Faculdade de Ciências Médicas, Campinas, 2004.

CONCEIÇÃO, M. et al. Levantamento da situação de saúde mental e uso de ansiolíticos e antidepressivos por acadêmicos dos cursos gerais. Revista da Universidade do Vale do Rio Verde, v.15, n.1, p. 489-496, 2017.

COUTINHO, D. C. M.; SANTOS, R. O trabalho do/da assistente social na Saúde Mental: atribuições privativas e competências profissionais em debate. Revista EDUC, Rio de Janeiro, v. 3, n. 1, p. 93-105, 2016.

DYNIEWICZ, A. M. Metodologia da pesquisa em saúde para iniciantes. 2. ed. São Caetano do Sul (SP): Difusão Cultural, 2009.

FERNANDES, K. J. S. S. et al. Relato de experiência: vivências de extensão na comunidade. Rev. Ciênc. Ext., v.12, n.1, p.97-104, 2016.

FERNANDES, T. F. et al. Uso de substâncias psicoativas entre universitários brasileiros: perfil epidemiológico, contextos de uso e limitações metodológicas dos estudos.

Cadernos de Saúde Coletiva, v. 25, n. 4, p. 498-507, 2018.

FERRO, L. F. et al. Interdisciplinaridade e intersetorialidade na Estratégia Saúde da Família e no Núcleo de Apoio à Saúde da Família: potencialidades e desafios. O Mundo da Saúde, [s.1.], v. 38, n. 2, p.129-138, 30 jun. 2014. .

FÓRUM Nacional de Pró-reitores de Assuntos Comunitários e Estudantis FONAPRACE. Mapeamento da assistência praticada nas IES. 2015.

GULLIVER, A. et al. University staff mental health literacy, stigma and their experience of students with mental health problems. Journal of Further and Higher Education, [s.1.], v. 43, n. 3, p.434-442, 31 ago. 2017.

KWAN, M. Y. et al. Patterns of multiple health risk-behaviours in university students and their association with mental health: application of latent class analysis. Health promotion and chronic disease prevention in Canada: research, policy and practice, v. 36, n. 8, p. 163, 2016. 
LANTYER, A. D. S. et al. Ansiedade e Qualidade de Vida entre Estudantes Universitários Ingressantes: Avaliação e Intervenção. Revista Brasileira de Terapia Comportamental e Cognitiva, v. 18, n. 2, p. 4-19, 2018.

LEITE, A. F.; NOGUEIRA, J. A. D. Fatores condicionantes de saúde relacionados ao trabalho de professores universitários da área da saúde: uma revisão integrativa. Revista Brasileira de Saúde Ocupacional, v. 42, n. 0, p. 1-15, 2017.

MINAYO, M. C. de S. O desafio do conhecimento: pesquisa qualitativa em saúde. São Paulo: Hucitec/ABRASCO, 2000. 269p.

MINISTÉRIO DA SAÚDE. Práticas Integrativas e Complementares (PICS): quais são e para que servem. Ministério da Saúde, 2019. Disponível em: www.saude.gov.br/saudede-a-z/praticas-integrativas-e-complementares.

MENDES, A. C.et al. Educação em enfermagem de saúde mental e psiquiatria no curso de licenciatura em enfermagem. SMAD Revista Eletrônica Saúde Mental Álcool e Drogas, v. 14, n. 2, p. 73-83, 2018.

NASCIMENTO, M. G. G.et al. O processo de trabalho do enfermeiro na promoção da saúde mental: análise reflexiva. Revista de Enfermagem do Centro-oeste Mineiro, [s.1.], v. 7, p.01-06, 20 dez. 2017. Disponível em:

http://dx.doi.org/10.19175/recom.v7i0.2097.

NOGUEIRA, M. J.; SEQUEIRA, C. A saúde mental em estudantes do ensino superior: relação com o género, nível socioeconómico e os comportamentos de saúde. Revista Portuguesa de Enfermagem de Saúde Mental, v. 5, n. spe5, p. 51-56, 2017.

SARACENO, B.; ASIOLI, F.; TOGNONI, G. Manual de Saúde Mental: 3. ed. São Paulo: Hucitec, 2001.

SILVA, B. P. da et al. Transtornos mentais comuns e consumo de bebida alcoólica e tabaco entre estudantes de enfermagem de uma universidade pública na Amazônia Ocidental brasileira. SMAD. Revista Eletrônica Saúde Mental Álcool e Drogas, v. 10, n. 2, p. 93, 2015.

STREETER C.C. et al. Treatment of Major Depressive Disorder with Iyengar Yoga and Coherent Breathing: A Randomized Controlled Dosing Study. J Atern Complement Med., v.23, n.3, p.201-207, mar. 2017.

TREMBLAY, Dominique et al. Effects of interdisciplinary teamwork on patient-reported experience of cancer care. BMC health services research, v. 17, n. 1, p. 218, 2017. 